\title{
La place de la compétence paraphrastique dans le Cadre européen commun de référence pour les langues
}

\author{
Alexandra Tsedryk \\ alexandra.tsedryk@msvu.ca
}

Mount SAINT Vincent University

\begin{abstract}
Résumé
Savoir produire des phrases synonymes, en se servant de divers moyens linguistiques, est indispensable pour un apprenant L2. L'objectif de cet article est de définir la compétence paraphrastique $(\mathrm{CP})$ et de déterminer sa place dans les descripteurs du Cadre européen commun de référence pour les langues (CECRL). Nous nous intéressons aux apprenants des niveaux B1-B2 du CECRL. L'apprenant avancé possède des connaissances grammaticales assez développées, mais il commet des erreurs lexicales et éprouve des difficultés à reformuler son discours.

Bien que les descripteurs du CECRL fassent référence à la $C P$ implicitement, une description détaillée de cette compétence cruciale fait défaut. Nous proposons cette description, en nous basant sur les critères quantitatifs et qualitatifs définis dans notre étude empirique examinant les stratégies de reformulation des apprenants avancés de français L2. Le cadre théorique adopté est la Théorie Sens-Texte qui porte une attention particulière à la paraphrase et possède des outils formels de sa description.

Mots-clés : compétence paraphrastique, apprenant avancé, descripteurs du CECRL
\end{abstract}

\begin{abstract}
The ability to produce synonymous sentences by various linguistic means is very important for L2 learners. The goal of this article is to define paraphrasing competence and identify its place among descriptors of the Common European Framework of Reference for Languages (CEFR). The focus is on CEFR B1 and B2 level learners. Advanced learners have fairly sophisticated grammatical skills, but still make lexical errors and experience difficulties in reformulating discourse.

Although CEFR descriptors implicitly refer to paraphrasing competence, a detailed description of this crucial competence is lacking. We have therefore proposed such a description based on quantitative and
\end{abstract}


qualitative criteria used in our empirical study, which examines the reformulation strategies used by advanced learners of French L2. The theoretical framework for this study is Meaning-Text Theory, which pays special attention to paraphrasing and has formal tools to describe it.

Key words: paraphrasing competence, advanced learner, CEFR descriptors

\section{Introduction}

Les cadres de référence pour les langues existants, tels que le Cadre européen commun de référence pour les langues (CECRL) ou les Niveaux de compétence linguistique canadiens (NCLC), ont pour objectif de décrire les compétences linguistiques variées des apprenants à des périodes d'apprentissage différentes. Ainsi, leurs utilisateurs potentiels (enseignants, étudiants, auteurs de manuels et évaluateurs d'examens) peuvent s'en servir afin de prendre des décisions éclairées et stratégiques selon leurs besoins.

Parmi une multitude de compétences linguistiques, nous choisissons la compétence paraphrastique $(\mathrm{CP})$, c'est-à-dire la capacité de reformuler une phrase de façon à en produire une autre ayant à peu près le même sens. Savoir reformuler est important pour mener à bien plusieurs tâches écrites et orales. En particulier, au niveau des études postsecondaires, cette compétence est requise dans la production des résumés, des comptes rendus, des synthèses de documents et des traductions.

Bien que l'intérêt pour la description de la CP soit évident dans les recherches récentes (Uemlianin, 2000; Keck, 2006; McInnis, 2009; McCarthy, Guess et McNamara, 2009; Martinot, 2010; Milićević et Tsedryk, 2011; Martinot et Gerolimich, 2012; Khrismawan et Widiati, 2013), les descripteurs des différents niveaux de cette compétence font défaut.

Les études précédentes ont examiné la compétence paraphrastique sous des angles variés. Ainsi, Uemlianin (2000) étudie le lien entre la capacité de paraphraser et la compréhension des textes. Selon cette étude, le locuteur ayant reformulé un passage compliqué d'un texte réussit à mieux en saisir le sens. L'approche d'analyse utilisée est l'attribution de points selon 25 critères préétablis et la comparaison des notes des participants.

Martinot et Gerolimich (2012) ainsi que Martinot (2010) ont pour objectif de décrire le lien entre la CP et l'acquisition de la langue maternelle chez les enfants. L'approche dans l'analyse des paraphrases produites par des enfants de différentes tranches d'âge (de 4 à 10 ans) est intéressante du point de vue méthodologique, car les auteurs décrivent en détail les stratégies de paraphrasage employées. Cependant, ces études visent l'acquisition d'une langue par des enfants, alors que nous cherchons à éclaircir le processus d'apprentissage 
du paraphrasage chez les adultes.

D'autres études, telles que Keck (2006), McInnis (2009), Khrismawan et Widiati (2013), entre autres, portent sur le rapport entre la compétence paraphrastique et le plagiat. Au niveau universitaire, les étudiants sont souvent appelés à résumer des textes d'auteurs en leurs propres mots, ce qui présuppose une bonne capacité à reformuler. Il en va de même pour les apprenants de langues étrangères à des niveaux avancés. Or, Keck constate l'insuffisance des moyens paraphrastiques chez les apprenants d'une L2 au niveau avancé par comparaison avec les locuteurs natifs. Même si Keck élabore une typologie de paraphrases (allant de quasi-copie jusqu'à révision importante), celle-ci ne prend en compte que le nombre de mots directement recopiés du texte source et ne permet pas de proposer des niveaux de CP bien décrits. Ayant constaté le manque de descripteurs de cette compétence très importante en L1 et en L2 dans les études mentionnées ci-dessus, nous procédons à l'examen du cadre de référence pour les langues existant, le CECRL. L'objectif de cet article est donc de cerner la place de la compétence paraphrastique dans ce cadre et de proposer une piste pour la création de descripteurs basés sur notre étude empirique menée récemment.

Cet article est organisé de la façon suivante. D'abord, nous résumons les connaissances nécessaires pour paraphraser en langue seconde (section 2). Ensuite, nous considérons la place de la CP dans les descripteurs du CECRL (section 3) et nous proposons un nouveau modèle d'évaluation de la $\mathrm{CP}$ en français L2 (section 4), suivi d'une conclusion (section 5).

\section{Définition de la compétence paraphrastique}

Les paraphrases sont des phrases sémantiquement équivalentes ou quasi équivalentes, mais différentes du point de vue lexical et syntaxique, comme celles en (1).

(1) a. Paul s'enthousiasme pour l'art dramatique.

b. Paul manifeste beaucoup d'enthousiasme pour le théâtre.

c. Paul apprécie grandement le théâtre.

d. Le thêâtre est la passion de Paul.

Afin de produire des paraphrases, le locuteur a besoin de connaissances linguistiques, telles que des connaissances lexicales, grammaticales et discursives, et de connaissances extralinguistiques centrées sur la situation de communication. L'apprenant doit savoir utiliser ces connaissances d'une façon efficace dans le discours. En d'autres termes, il lui faut produire une phrase qui conserve le sens de la phrase de départ, utiliser des moyens paraphrastiques de nature différente et choisir parmi plusieurs variantes paraphrastiques celle 
qui convient le mieux à sa tâche de communication. En prenant en considération les types de connaissances nécessaires pour faire du paraphrasage, Tsedryk (2013, pp. 168-169) propose la définition suivante de la compétence paraphrastique :

La compétence paraphrastique d'un locuteur, c'est l'ensemble des connaissances et des habiletés qui lui permettent de produire et d'utiliser efficacement dans le discours, dans différents contextes de communication linguistique, des paraphrases de types variés et d'un haut niveau de correction.

Observons maintenant comment la $\mathrm{CP}$ est décrite dans notre document de référence, le CECRL.

\section{Descripteurs du CECRL et la compétence paraphrastique}

Le Cadre adopte une structure arborescente des niveaux d'apprentissage. Ainsi, les trois niveaux généraux A (élémentaire), B (indépendant) et C (expérimenté) sont subdivisés chacun en deux sous-niveaux, comme le montre la figure 1.

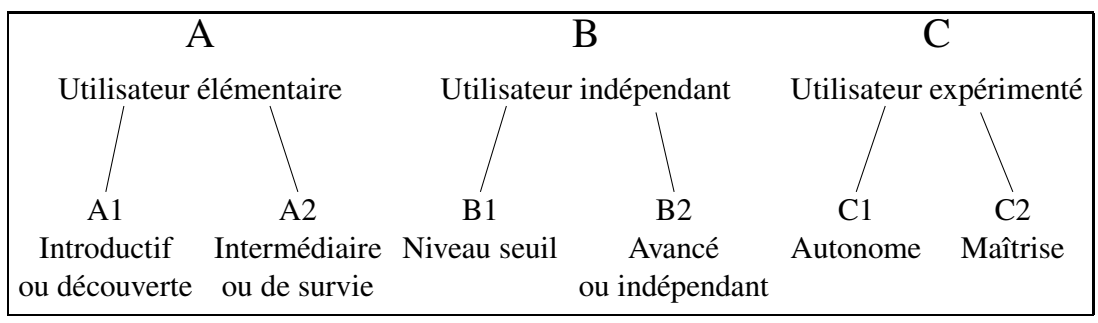

\section{FIGURE 1}

Structure arborescente du CECRL (2001, p. 25)

Cette structure permet de décrire en détail les différentes compétences langagières. Les apprenants qui nous intéressent se situent aux niveaux B1 et B2. Les niveaux $\mathrm{C} 1$ et $\mathrm{C} 2$ correspondent à une véritable maîtrise de la langue. Les descripteurs des niveaux $\mathrm{C} 1$ et $\mathrm{C} 2$ font référence à la capacité de reformuler le discours (CECRL, 2001, p. 28) :

C1 : [l'apprenant a] une bonne maitrise d'une grande gamme de discours parmi lesquels il peut choisir la formulation lui permettant de s'exprimer clairement ...

C2: [l'apprenant m]ontre une grande souplesse dans la reformulation des idées sous des formes linguistiques différentes...

Examinons maintenant les niveaux B1 et B2, qui sont les plus pertinents pour notre étude. Au niveau B2, l'apprenant a déjà acquis les structures grammaticales qui lui permettent de contrôler sa production et de s'exprimer presque 
sans erreurs. Son vocabulaire est assez varié puisqu'il est capable de parler de sujets différents sans lacunes lexicales apparentes. Quant au niveau B1, on mentionne la difficulté de l'apprenant à reformuler des phrases d'une façon idiomatique. Cet apprenant manque de souplesse langagière qui rendrait son discours aussi naturel et spontané que celui d'un locuteur natif. À ce niveau, l'individu a assez de moyens linguistiques pour "s'en sortir avec quelques hésitations et quelques périphrases ${ }^{1} \gg($ CECRL, 2001, p. 87) et combler ses lacunes en vocabulaire.

Nous ne trouvons qu'une seule mention directe sur le paraphrasage dans le tableau « Traiter un texte», pour le niveau seuil B1 (CECRL, 2001, p. 77), indiquant qu'un apprenant de ce niveau « [p]eut paraphraser simplement de courts passages écrits en utilisant les mots et le plan du texte» (p. 77). L'habileté à paraphraser est mentionnée néanmoins dans la documentation des épreuves de langues. Ainsi, le Manuel pour relier les examens de langues du CECRL (2009), préparé par la Division des politiques linguistiques du Conseil de l'Europe, contient un tableau de référence pour l'évaluation de la production écrite. Nous y retrouvons la même mention sur le paraphrasage que celle qui figure dans le CECRL, selon laquelle l'apprenant au niveau B1 est capable de paraphraser en empruntant les mots du texte et de «collationner des éléments d'information issus de sources diverses et [de] les résumer pour quelqu'un d'autre » (p. 77). En d'autres termes, l'apprenant au niveau B1 est en manque de moyens de paraphrasage, car il se sert des mots du texte source pour résumer un passage. Au niveau B2, l'apprenant peut résumer un large éventail de textes factuels. Le reste des descriptifs porte surtout sur les types de textes que les apprenants des niveaux B2-C2 sont en mesure de résumer, mais n'offre pas de détails sur les types d'opérations de reformulation mises en œuvre.

Malgré le fait que les références à la CP sont presque absentes du CECRL, l'importance de la capacité de paraphraser est mise en évidence dans les épreuves écrites des examens Diplôme d'études en langue française (DELF) et Diplôme approfondi de langue française (DALF). Ainsi, dans l'épreuve du DALF 2007 pour le niveau C1 (conçue par le Centre international d'études pédagogiques [CIEP]), en production écrite, on trouve un exercice de synthèse de documents. L'apprenant doit rédiger un texte de 220 mots afin de présenter, d'une façon concise et claire, l'information contenue dans deux articles de presse. Dans la description de l'exercice, on demande à l'apprenant de ne pas

\footnotetext{
${ }^{1}$ Il convient de ne pas confondre le terme périphrase et le terme paraphrase que nous utilisons dans cet article. D'après Le Petit Robert (édition de 2013), la périphrase (ling.) est « un groupe de mots synonyme d'un seul mot», par exemple, femelle du cheval pour jument. La périphrase dans le CECRL est considérée, entre autres, comme une technique de compensation dont un apprenant se sert en cas d' «inadéquation de la compétence communicative » (2001, p. 75).
} 
recopier les phrases : «vous pouvez bien entendu réutiliser les «mots clefs » des documents, mais non des phrases ou des passages entiers » (CIEP, 2007b, p. 8). Cet exercice sert donc à évaluer, entre autres, la compétence paraphrastique de l'apprenant. Nous trouvons dans la grille d'évaluation de l'examinateur des extraits de descripteurs présentés dans la figure 2 (CIEP, 2007a, p. 1).

\begin{tabular}{|c|}
\hline $\begin{array}{c}\text { Compétence lexicale, étendue et maitrise du vocabulaire } \\
\text { Dispose d'un vaste répertoire lexical lui permettant de } \\
\text { reformuler sans effort apparent. }\end{array}$ \\
\hline Compétence grammaticale, élaboration des phrases/souplesse \\
Dispose d'une variété de structures lui permettant de varier la formulation. \\
\hline
\end{tabular}

FIGURE 2

Extraits de la grille d'évaluation en production écrite DALF C1

Si l'apprenant ne réussit pas à reformuler les phrases en ses propres mots, l'évaluateur doit le pénaliser. Voici l'extrait de la consigne pour l'examinateur: «Dans le cas où un candidat reprendrait, sans les remanier, des passages entiers des documents (plus de 3/4 du texte final), les notes à attribuer pour les critères « compétence lexicale » et « compétence grammaticale » seraient mises à 0 » (CIEP, 2007a, p. 1).

Le tableau 1 contient un résumé des compétences langagières des niveaux B1-C2 du CECRL (pp. 87-88). En gras, nous avons marqué les passages relatifs à la compétence paraphrastique.

Après avoir étudié les descripteurs des niveaux de compétences du CECRL, nous pouvons conclure que la compétence paraphrastique est sous-entendue dans cet ouvrage de référence, mais n'y figure pas d'une façon explicite. Nous proposerons, dans la section suivante, nos propres descripteurs pour les niveaux de CP.

\section{Notre approche}

Notre réflexion sur la description de la CP naît d'une étude que nous avons menée récemment auprès des apprenants du français L2 $(n=40)$. Le but de cette étude était d'établir la mesure dans laquelle les locuteurs non natifs, par comparaison avec les locuteurs natifs, comprennent la notion de paraphrase et de déterminer les stratégies de reformulation dont ils disposent et les sortes de difficultés qu'ils éprouvent lorsqu'ils reformulent des énoncés. Les apprenants du français L2, étudiants de troisième et quatrième années se spécialisant en français dans deux universités canadiennes, avaient en moyenne 22 ans. Le groupe témoin, locuteurs natifs à peu près du même âge, comprenait 40 étudiants de cégeps et d'universités québécoises. Le test de paraphrasage que nous 


\section{TABLEAU 1}

Niveaux communs de compétences langagières du CECRL (2001, pp. 87-88)

\begin{tabular}{ll}
\hline \hline Étendue linguistique générale \\
\hline B1 & $\begin{array}{l}\text { Possède suffisamment de moyens } \\
\text { linguistiques pour s'en sortir avec }\end{array}$ \\
& quelques hésitations et quelques pé- \\
& riphrases sur des sujets tels que la fa- \\
& mille, les loisirs et les centres d'intérêt, \\
& le travail, les voyages et l'actualité ...
\end{tabular}

B2 Possède une gamme assez étendue de langue pour pouvoir faire des descriptions claires, exprimer son point de vue et développer une argumentation sans chercher ses mots de manière évidente et en utilisant des phrases complexes.

\section{Étendue du vocabulaire}

Possède un vocabulaire suffisant pour s'exprimer à l'aide de périphrases sur la plupart des sujets relatifs à sa vie quotidienne tels que la famille, les loisirs et les centres d'intérêt, le travail, les voyages et l'actualité.

Possède une bonne gamme de vocabulaire pour les sujets relatifs à son domaine et les sujets les plus généraux. Peut varier sa formulation pour éviter de répétitions fréquentes, mais des lacunes lexicales peuvent encore provoquer des hésitations et l'usage de périphrases.

C1 Peut choisir la formulation appropriée dans un large répertoire de discours pour exprimer sans restriction ce qu'il/elle veut dire.

Possède une bonne maîtrise d'un vaste répertoire lexical lui permettant de surmonter facilement les lacunes par des périphrases avec une recherche peu apparente d'expressions et de stratégies d'évitement. Bonne maitrise d'expressions idiomatiques et familières.

C2 Peut exploiter la maîtrise exhaustive et fiable d'une gamme très étendue de discours pour formuler précisément sa pensée, insister, discriminer et lever l'ambiguïté ...

Possède une bonne maîtrise d'un vaste répertoire lexical d'expressions idiomatiques et courantes avec la conscience du niveau de connotation sémantique.

avons fait passer à tous les participants comportait cinq phrases de départ. Les étudiants devaient proposer pour chacune trois reformulations. Les phrases de départ se prêtant à de nombreuses reformulations étaient accompagnées d'un contexte minimal afin de préciser le sens des lexies employées. Bien que le concept de paraphrase ait été expliqué succinctement avant la collecte des données, les participants n'ont pas obtenu de formation explicite sur la notion et les stratégies de paraphrasage. Les phrases obtenues $(n=583)$, qui constituent notre corpus, ont été analysées selon trois critères : le sens (sens maintenu ou modifié); les opérations de paraphrasage effectuées (sémantiques, lexicales 
ou syntaxiques); et la correction grammaticale et stylistique (types d'erreurs commises). Un lecteur intéressé peut trouver plus de détails sur la méthodologie de collecte de données et les instruments de recherche dans Tsedryk (2013, pp. 82-90) et Tsedryk (2014a).

\section{1. Évaluation de la compétence paraphrastique}

Le modèle que nous avons utilisé pour évaluer la CP comporte deux axes : l'axe quantitatif et l'axe qualitatif (Tsedryk, 2014b). Dans cet article, nous nous concentrons sur l'axe qualitatif, comme le montre la figure 3 (p. 70).

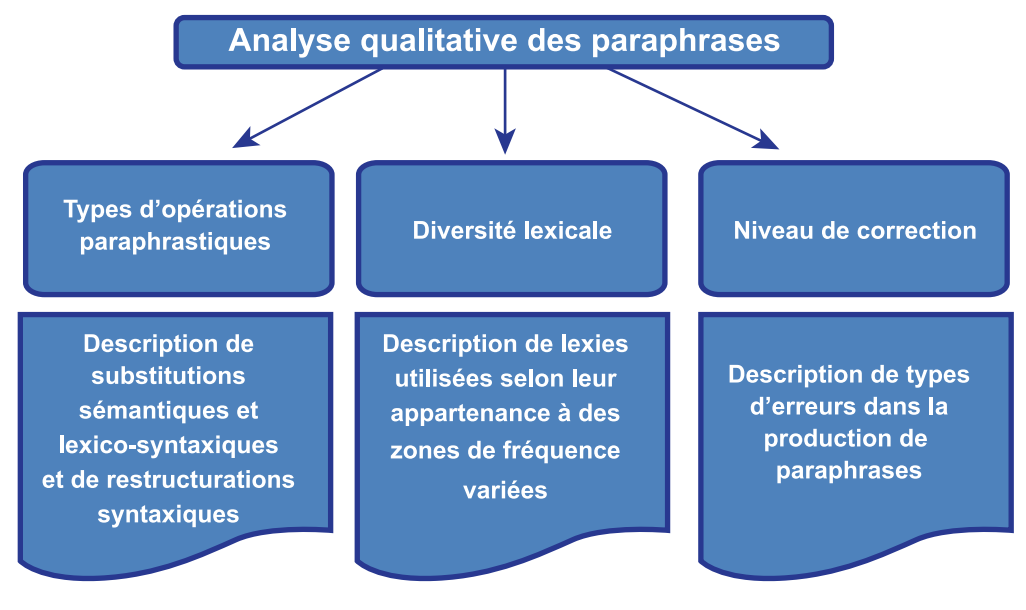

FIGURE 3

Analyse qualitative de la compétence paraphrastique

Nous avons établi quatre niveaux de CP en nous servant de l'analyse statistique, la méthode des k-moyennes ${ }^{2}$, et avons procédé à l'analyse qualitative d'un échantillon des paraphrases produites par des participants de chaque niveau obtenu. Pour ce faire, nous avons identifié trois participants dans chaque groupe dont les productions paraphrastiques ont obtenu des résultats proches des moyennes de chaque niveau particulier. Les trois critères suivants ont guidé notre analyse de données : (a) les types de substitutions paraphrastiques effectuées par les participants, (b) la fréquence des lexies utilisées selon leur appartenance à des zones de fréquence variées, et (c) les types d'erreurs commises lors du paraphrasage.

En premier lieu, nous avons identifié les opérations paraphrastiques utili-

\footnotetext{
${ }^{2}$ Faute de place dans cet article, nous ne présentons pas notre démarche pour l'évaluation quantitative des paraphrases. Les détails peuvent être consultés dans Tsedryk (2013, pp. 171-180).
} 
sées par les participants, en nous appuyant sur la typologie des paraphrases proposée dans le cadre de la théorie Sens-Texte (Mel'čuk 1992, Milićević 2007). On distingue, grosso modo, des opérations sémantiques, lexico-syntaxiques et syntaxiques. Par exemple, les paraphrases produites par l'un des participants au niveau 1 du corpus témoignent d'un usage assez fréquent des opérations syntaxiques de passivisation $(2,3 \mathrm{a}, 3 \mathrm{c})$, menant quelquefois à des phrases erronées, comme en (2a) ${ }^{3}$. Ce même participant a utilisé la relativisation (3) pour produire des paraphrases.

(2) a. [Marc a toujours beaucoup de questions pour le professeur de français.] *Le professeur de français est demandé plusieurs questions toujours par Marc.

b. [Julie admire énormément l'auteur de ce roman historique.] L'auteur de ce roman historique est admiré énormément par Julie.

(3) a. [Julie admire énormément l'auteur de ce roman historique.] Ce roman historique est écrit par l'auteur que Julie admire énormément.

b. L'auteur que Julie admire énormément a écrit ce roman historique.

c. [Le professeur a donné un bon conseil à l'étudiant.] Un bon conseil, qui a été donné par le professeur, est reçu par l'étudiant.

Quant aux opérations lexico-syntaxiques complexes, au niveau 1 de la CP, elles sont rares. Ce sont des opérations comportant la substitution d'une lexie par une autre et accompagnées de restructurations syntaxiques. Les substitutions simples qui prévoient la substitution d'une lexie par une autre sont plus nombreuses que les substitutions complexes au niveau 1 de la CP. Par exemple, dans la phrase (3c) l'apprenant a utilisé la conversion lexicale $X$ donne un conseil à $Y \sim Y$ reçoit un conseil de $X$. Ce participant a aussi utilisé une opération sémantique portant sur la présentation du contenu informatif de la phrase. Il s'agit d'un changement dans l'organisation thémo-rhématique : le thème de la phrase de départ est «le professeur», alors que celui de la paraphrase correspondante est « un bon conseil».

En deuxième lieu, nous avons analysé la fréquence des lexies utilisées dans les paraphrases en nous servant du logiciel Vocabprofile (Cobb, 2010) ${ }^{4}$. Nous avons déterminé le nombre de lexies appartenant à l'une des quatre zones de fréquence $(1 \mathrm{~K}$ : premier millier de mots les plus fréquents, $2 \mathrm{~K}$ : deuxième millier, $3 \mathrm{~K}$ : troisième millier; MHL : mots hors liste, qui n'apparaissent dans aucune de trois premières zones de fréquence) dans les paraphrases produites par les participants.

\footnotetext{
${ }^{3}$ Les phrases de départ sont indiquées entre crochets.

${ }^{4}$ Les listes de fréquence ont été compilées par T. Selva (Verlinde et Selva, 2001) et par G. Jones et R. Goodfellow (Jones, 2001) et adaptées par T. Cobb (www.lextutor. ca).
} 
Enfin, nous avons analysé les paraphrases produites par les étudiants du point de vue de correction grammaticale et lexicale. Par exemple, pour le niveau 1 de la $\mathrm{CP}$, nous pouvons constater les erreurs lexicales de collocation, *demander des questions (2a), et les erreurs syntaxiques, comme l'emploi du passif indirect influencé par la langue maternelle (2a) et le mauvais ordre des mots (2a).

\subsection{Descripteurs de la compétence paraphrastique}

Nous avons analysé 107 paraphrases proposées par les 12 participants choisis. En appliquant les critères qualitatifs décrits dans la section précédente, nous avons pu établir les caractéristiques propres à chaque niveau de CP. Nous résumons celles du niveau 1 (élémentaire) et du niveau 4 (supérieur) dans le tableau 2.

Remarquons que les niveaux de CP que nous identifions correspondent au niveau seuil du CECRL (B1) et aux niveaux plus avancés : le locuteur peut déjà s'exprimer dans la langue étudiée. Autrement dit, il possède les connaissances linguistiques nécessaires pour formuler sa pensée. Le niveau 4 de CP correspond au niveau de la plupart des membres du groupe de contrôle; les locuteurs natifs ayant une bonne aptitude à paraphraser ont été placés à ce niveau. Aucun de nos participants non natifs ne s'est classé au niveau 4 de CP.

En examinant les descripteurs du CECRL et les nôtres, nous remarquons des similitudes à chaque niveau. Prenons, par exemple, le niveau B1 du CECRL (nous soulignons). À ce niveau, l'apprenant est en mesure de produire des textes familiers, mais il a des difficultés à exprimer une pensée complexe. En d'autres termes, il maîtrise des lexies courantes et il est capable de produire des phrases syntaxiquement simples. Cependant, les constructions multipropositionnelles dont on se sert pour exprimer une pensée complexe représentent pour lui une difficulté. De plus, on observe une nette influence de sa langue maternelle sur ses productions. Nous trouvons des ressemblances avec notre descripteur de la CP pour le niveau élémentaire : un apprenant produit des phrases lexicalement et structurellement simples, en utilisant les lexies de haute fréquence et en faisant des erreurs influencées par sa LI.

\section{Conclusion}

Dans cet article, nous avons amorcé une réflexion sur la description qualitative de la compétence paraphrastique d'un locuteur. Nous avons étudié les descripteurs des niveaux avancés dans le cadre de référence connu internationalement, le Cadre européen commun de référence pour les langues. La capacité de reformuler un discours y est sous-entendue aux niveaux B1-C2, mais elle n'y est pas explicitement décrite. Or, c'est en examinant les compétences particulières dans les cadres de référence et en proposant des activités qui ciblent ces com- 
TABLEAU 2

Caractéristiques des niveaux 1 et 4 de compétence paraphrastique

\begin{tabular}{|c|c|c|}
\hline $\begin{array}{l}\text { Niveaux de } \\
\text { compétence } \\
\text { paraphrastique }\end{array}$ & 1 : niveau élémentaire & 4 : niveau supérieur \\
\hline \multirow[t]{3}{*}{$\begin{array}{l}\text { Opérations para- } \\
\text { paraphrastiques }\end{array}$} & $\begin{array}{l}\text { L'apprenant: } \\
\text { Utilise des phrases lexicale- } \\
\text { ment et structurellement } \\
\text { simples. Utilise souvent des } \\
\text { opérations syntaxiques de } \\
\text { passivisation et de relativi- } \\
\text { sation et change l'ordre des } \\
\text { mots. }\end{array}$ & $\begin{array}{l}\text { L'apprenant : } \\
\text { Se sert d'opérations } \\
\text { syntaxiques diversifiées et } \\
\text { complexes. }\end{array}$ \\
\hline & $\begin{array}{l}\text { Fait des substitutions } \\
\text { synonymiques simples. } \\
\text { N'utilise pas d'opérations } \\
\text { lexico-syntaxiques/ } \\
\text { syntaxiques complexes. }\end{array}$ & $\begin{array}{l}\text { Possède un vaste répertoire } \\
\text { d'opérations lexico- } \\
\text { syntaxiques et lexico- } \\
\text { syntaxiques/syntaxiques } \\
\text { complexes. }\end{array}$ \\
\hline & $\begin{array}{l}\text { Utilise des décompositions } \\
\text { sémantiques entraînant } \\
\text { souvent des expressions } \\
\text { maladroites. }\end{array}$ & $\begin{array}{l}\text { Utilise des opérations } \\
\text { sémantiques diverses en } \\
\text { produisant des paraphrases } \\
\text { correctes. }\end{array}$ \\
\hline $\begin{array}{l}\text { Diversité } \\
\text { lexicale }\end{array}$ & $\begin{array}{l}\text { Utilise des lexies de haute } \\
\text { fréquence }(1 \mathrm{~K}-2 \mathrm{~K}) \text { dans les } \\
\text { substitutions synonymiques. }\end{array}$ & $\begin{array}{l}\text { Possède un choix de lexies } \\
\text { très varié dans les } \\
\text { substitutions lexico- } \\
\text { syntaxiques. L'utilisation des } \\
\text { lexies de moindre fréquence } \\
\text { ( } 3 \mathrm{~K}-\mathrm{MHL} \text { ) se généralise. }\end{array}$ \\
\hline $\begin{array}{l}\text { Niveau de } \\
\text { correction }\end{array}$ & $\begin{array}{l}\text { Commet des erreurs lexicales } \\
\text { et syntaxiques influencées par } \\
\text { la langue maternelle. Les } \\
\text { erreurs morphologiques sont } \\
\text { évidentes. }\end{array}$ & $\begin{array}{l}\text { A un haut niveau de } \\
\text { correction grammaticale. Les } \\
\text { erreurs sont occasionnelles. }\end{array}$ \\
\hline
\end{tabular}

pétences que les enseignants de langues sont en mesure d'accroître l'efficacité de l'enseignement. Quels concepts liés à la production de paraphrases faut-il enseigner? Quels types de paraphrases posent le plus de difficultés aux apprenants avancés du français L2 ? Ces derniers comprennent-ils la notion même de paraphrase ? Quelles stratégies de reformulation s'avèrent les plus productives? Quelles connaissances sous-jacentes au paraphrasage faut-il acquérir? Ces questions restent actuellement sans réponses dans le CECRL.

Puisqu'une description explicite et détaillée de la CP aux niveaux avancés d'apprentissage fait défaut dans le Cadre, il est difficile, voire impossible, pour 
les pédagogues de mettre en place des activités qui stimuleraient le développement de cette compétence cruciale. Nous avons donc essayé de combler cette lacune, en adoptant une approche structurée ancrée dans une théorie linguistique et basée sur une étude empirique. Notre approche permet de cerner les difficultés des apprenants avancés d'une L2 lors du processus de reformulation. Ainsi, les descripteurs proposés permettent d'élargir le spectre d'activités portant sur le paraphrasage dans les salles de classe. Par exemple, ces activités comprendraient des exercices sur la notion de paraphrase, les stratégies de paraphrasage sémantiques, lexico-syntaxiques et syntaxiques, l'utilisation de lexies de moindre fréquence lors des reformulations, l'usage des connaissances de relations lexicales entre les lexies de la langue, et l'analyse contrastive des paraphrases en français et en d'autres langues. Enfin, les activités sur l'analyse d'erreurs dues à l'influence de la langue maternelle des apprenants contribueraient au développement de la $\mathrm{CP}$ au niveau avancé d'études.

En nous fiant à notre intuition, nous supposons que le niveau 1 (élémentaire) de CP correspondrait au niveau B1 du CECRL (niveau seuil) et le niveau 4 (supérieur), au niveau C2. Notons toutefois qu'il s'agit d'une comparaison préliminaire, car une recherche portant sur l'usage parallèle des tests, tels que notre test de reformulation et les épreuves DELF ou DALF, est indispensable pour tirer des conclusions définitives quant à la correspondance entre ces niveaux. Une piste de recherche future consistera à déterminer plus précisément le lien entre nos descripteurs de compétence paraphrastique et les descripteurs de compétences linguistiques du CECRL.

\section{Références}

Centre international d'études pédagogiques (CIEP). 2007a. DALF C1. Grille d'évaluation de la production écrite C1, pp. 1-2. Sèvres, France. Consulté le 8 juin 2015. Disponible à : piaf.ciep.fr/_DELF_DALF/Documents/C1_Grille_PE.pdf.

Centre international d'études pédagogiques (CIEP). 2007b. DALF C1. Document réservé au candidat - épreuves collectives, pp. 1-13. Sèvres, France.

Cobb, T. 2010. VocabProfile, The Compleat Lexical Tutor, v. 6.2), French VP_Fr (v.2.7). Consulté le 20 avril 2014. Disponible à : www.lextutor.ca.

Conseil de l'Europe. 2001. Un cadre européen commun de référence pour les langues : apprendre, enseigner, évaluer. Paris, Didier. Consulté le 27 août 2014. Disponible à : www.coe.int/t/dg4/linguistic/Source/Framework_FR.pdf.

Conseil de l'Europe. 2009. Relier les examens de langues au Cadre européen commun de référence pour les langues : apprendre, enseigner, évaluer (CECRL) : Un manuel. Strasbourg, Division des politiques linguistiques. Disponible à : www.coe.int/t/ dg4/linguistic/Source/ManualRevision-proofread-FINAL_fr.pdf.

Jones, G. 2001. Compiling French word frequency list for the VAT : A feasibility study. Document de travail. Consulté le 29 avril 2014. Disponible à : www.er.uqam.ca/nobel/ r21270/cgi-bin/F_webfreqs/glynn_jones.html. 
Keck, C. 2006. The use of paraphrase in summary writing : A comparison of L1 and L2 writers. Journal of Second Language Writing, 15, pp. 261-278.

Khrismawan, B. et U. Widiati. 2013. Students' perceptions about paraphrasing and their cognitive processes in paraphrasing. TEFLIN Journal, 24, pp. 135-157.

Martinot, C. 2010. Reformulation et acquisition de la complexité linguistique. Travaux de linguistique, 61, pp. 63-96.

Martinot, C. et S. Gerolimich. 2012. Acquisition de la compétence paraphrastique par des enfants francophones et italophones de 4 à 10 ans. Dans S. Cappello, M. Conenna et J.-P. Dubiet (dir.), La synonymie au-delà du lexique. Udine, Forum, pp. 191-224.

McCarthy, P.M., R.H. Guess et D.S. McNamara. 2009. The components of paraphrase evaluations. Behavior Research Methods, 41, pp. 682-690. Consulté le 20 avril 2014. Disponible à : 129.219.222.66/Publish/pdf/McCarthy_Guess_McNamara_2009_ paraphrase_evals.pdf.

McInnis, L. 2009. Analyzing English L1 and L2 paraphrasing strategies through concurrent verbal report and stimulated recall protocols. Thèse de maîtrise, Université de Toronto.

Mel'čuk, I. 1992. Paraphrase et lexique : la théorie Sens-Texte et le Dictionnaire explicatif et combinatoire. Dans I. Mel'čuk, N. Arbatchewsky-Jumarie, L. Iordanskaja et S. Mantha (dir.), Dictionnaire explicatif et combinatoire du français contemporain : recherches lexico-sémantiques III. Montréal, Les Presses de l'Université de Montréal, pp. 9-58.

Milićević, J. 2007. La paraphrase : modélisation de la paraphrase langagière. Bern, Peter Lang.

Milićević, J. et A. Tsedryk. 2011. Assessing and improving paraphrasing competence in FSL. Dans I. Boguslavsky et L. Wanner (dir.), Proceedings of the 5th International Conference on Meaning-Text Theory. Barcelone, Universitat Pompeu Fabra, pp. $175-185$.

Tsedryk, A. 2013. Didactique de la paraphrase : évaluation et développement de la compétence paraphrastique chez l'apprenant de français langue seconde. Thèse de doctorat, Dalhousie University, Halifax.

Tsedryk, A. 2014a. Un autre regard sur l'apprenant avancé : sa capacité de paraphraser. Dans F. Neveu, P. Blumenthal, L. Hriba, A. Gerstenberg, J. Meinschaefer et S. Prévost (eds.), $4^{e}$ Congrès mondial de linguistique française. Paris, EDP sciences, pp. 1189-1200. Consulté le 25 juillet 2014. Disponible à : dx.doi.org/10.1051/shsconf/ 20140801134.

Tsedryk, A. 2014b. Définition et évaluation de la compétence paraphrastique. Dans P. Balcom, L. Beaulieu, S. Kasparian, C. Potvin et M.-L. Ungureanu (dir.), Actes du 37e colloque annuel de l'Association de linguistique des provinces atlantiques (ACAAPLA). Moncton, Université de Moncton, pp. 58-75. Consulté le 8 juin 2015. Disponible à : www.unb.ca/fredericton/arts/departments/french/apla-alpa/pdfs/vol37_ 2013/pamapla-37.pdf

Uemlianin, I. 2000. Engaging text : Assessing paraphrase and understanding. Studies in Higher Education, 25, pp. 347-358. 
Verlinde, S. et T. Selva. 2001. Corpus-based versus intuition-based lexicography : Defining a word list for French learner's dictionary. UCREL Technical Paper, 13, pp. 594-598. Special issue : Proceedings of the Corpus Linguistics 2001 conference, P. Rayson, A. Wilson, T. McEnery, A. Hardie et S. Khoja (dir.). Lancaster, UK, University Centre for Computer Corpus Research on Language (UCREL). 